\title{
Structural and Functional Evolution of Vertebrate Neuroendocrine Stress Systems
}

\author{
Robert John Denver \\ Department of Molecular, Cellular and Developmental Biology, and Department of \\ Ecology and Evolutionary Biology, The University of Michigan, Ann Arbor, Michigan, USA
}

\begin{abstract}
The vertebrate hypothalamus-pituitary-adrenal (HPA; or interrenal) axis plays pivotal roles in animal development and in physiological and behavioral adaptation to environmental change. The HPA, or stress axis, is organized in a hierarchical manner, with feedback operating at several points along the axis. Recent findings suggest that the proteins, gene structures, and signaling pathways of the HPA axis were present in the earliest vertebrates and have been maintained by natural selection owing to their critical adaptive roles. In all vertebrates studied, the HPA axis is activated in response to stressors and is controlled centrally by peptides of the corticotropin-releasing factor (CRF) family of which four paralogous members have been identified. Signaling by CRF-like peptides is mediated by at least two distinct $G$ protein-coupled receptors and modulated by a secreted binding protein. These neuropeptides function as hypophysiotropins and as neurotransmitters/neuromodulators, influencing stress-related behaviors, such as anxiety and fear. In addition to modulating HPA activity and behavioral stress responses, CRF-like peptides are implicated in timing key life history transitions, such as metamorphosis in amphibians and birth in mammals. CRF-like peptides and signaling components are also expressed outside of the central nervous system where they have diverse physiological functions. Glucocorticoids are the downstream effectors of the HPA axis, playing essential roles in development, energy balance and behavior, and feedback actions on the activity of the HPA axis.
\end{abstract}

Key words: stress; neuroendocrine system; corticotropin-releasing factor; urocortin; evolution; corticosteroids

\section{Introduction}

The vertebrate neuroendocrine stress axis (hypothalamus-pituitary-adrenal or interrenal; hereafter the HPA axis) is an ancient physiological system that plays a pivotal role in mediating organismal responses to environmental change. In all vertebrates that have been studied, neurosecretory neurons in the hypothalamus synthesize neuropeptides of the corticotropin-releasing factor (CRF) family that act on the pituitary gland to stimulate the release of corticotropin (adrenocorti-

Address for correspondence: Dr. Robert J. Denver, 3065C Kraus Building, 830 North University Avenue, The University of Michigan, Ann Arbor, MI 48109-1048. Voice: +734-936-6625; fax: +734-647-0884. rdenver@umich.edu cotropic hormone; ACTH). Corticotropin is a small peptide hormone (39 amino acids) derived by proteolytic processing of the precursor protein proopiomelanocortin (POMC); it is secreted into the systemic circulation and binds to receptors expressed in adrenocortical cells to increase the biosynthesis of corticosteroids.

Corticosteroids are the primary "stress hormones" of vertebrates and in mammals have been classified into two groups, the glucocorticoids (GCs) and the mineralocorticoids, owing to their often distinct physiological functions. Such distinctions are less clear in nonmammalian species (e.g., in teleost fishes cortisol, a classical GC in humans, plays important roles in hydromineral balance). ${ }^{1}$ Corticosteroids have diverse actions in development,

Trends in Comparative Endocrinology and Neurobiology: Ann. N.Y. Acad. Sci. 1163: 1-16 (2009).

doi: 10.1111/j.1749-6632.2009.04433.x (C) 2009 New York Academy of Sciences. 
physiology, and behavior in vertebrate species. They are well known to influence development of the brain, lungs, and other organ systems; to mobilize stored energy, inhibit energy storage, and stimulate gluconeogenesis; to stimulate feeding behavior in order to replenish depleted energy stores following a stress response; and to influence learning and memory consolidation. A major, and perhaps primitive, role for corticosteroids is in osmoregulation. In all vertebrate species studied, corticosteroids have been shown to influence plasma ion concentrations. In teleost fishes cortisol (fish do not make the classical tetrapod mineralocorticoid aldosterone) stimulates sodium transport across epithelia of the gills, gut, and kidney and is essential for promoting seawater adaptation. ${ }^{1}$ Corticosteroids also function in negative feedback at the level of the brain and pituitary gland to inhibit the secretion of CRF and ACTH and thus return the system to basal following a stress response. A thorough discussion of the actions of corticosteroids is beyond the scope of this review, but the reader is directed to reviews cited throughout this paper for further information (and see Refs. 2-5).

The corticosteroids bind to two receptor subtypes belonging to the nuclear hormone receptor (NR) superfamily: the type I [the mineralocorticoid receptor (MR)] and the type II [the GC receptor (GR); Ref. 2]. The type I receptor has higher affinity than the type II for corticosteroids, is selective for aldosterone, and thus mediates actions of this mineralocorticoid on hydromineral balance (thus it is named MR); whereas the type II receptor plays a principal role in mediating GC actions during a stress response (thus it is named GR). The two receptors show region-specific expression in the brain, with the GR being more widely expressed than the MR. The differential affinities, specificities, and expression patterns have led to the hypothesis that the MR maintains basal activity of the HPA axis while the GR mediates negative feedback under rising GC concentrations in response to a stressor. ${ }^{2}$ There is also evidence for corticosteroid receptors (CRs) located in the plasma membrane that mediate rapid actions of these hormones. ${ }^{6}$

Studies of extant species show that many of the genes of the HPA axis are present in urochordates and cephalochordates; in fact, many stress-related genes predate the chordates, thus showing their ancient origins. ${ }^{7,8}$ Furthermore, the major functions of this axis in mediating developmental, physiological, and behavioral responses to environmental change are ancient features of the chordate lineage. ${ }^{8,9}$ Strong positive selection has maintained the structure and functions of this axis owing to the pivotal adaptive role that stress hormones play in individual survival.

\section{Evolutionary Origins of Corticosteroids and Their Receptors}

The biochemical pathways responsible for steroid biosynthesis and their function as signaling molecules are ancient and were likely present in the earliest eukaryotes. For example, plants and fungi synthesize steroids that are known to regulate development and reproduction. ${ }^{10,11}$ Plant steroid hormones (the brassinosteroids) signal via membrane receptor kinases. ${ }^{12}$ The NRs that mediate many of the actions of steroid hormones in animals are absent from plants and are estimated to have arisen over 1 billion years ago after the divergence of the metazoans and fungi but prior to the Cnidaria/Bilateria split. ${ }^{13}$

Although invertebrates synthesize a diversity of steroid hormones and have multiple NR genes, the capacity to synthesize 11- and 21hydroxylated steroids (the corticosteroids) appears to be unique to the vertebrates. This ability to synthesize corticosteroids likely arose prior to the origin of the jawed vertebrates (the gnathastomes) as cortisol, corticosterone, and 11-deoxycorticosterone (11-DOG) have been found in the blood of the Cyclostomata (hagfish and lamprey; reviewed by Ref. 14). Phylogenetic analysis of steroid receptors in basal vertebrates suggests that, prior to the evolution 


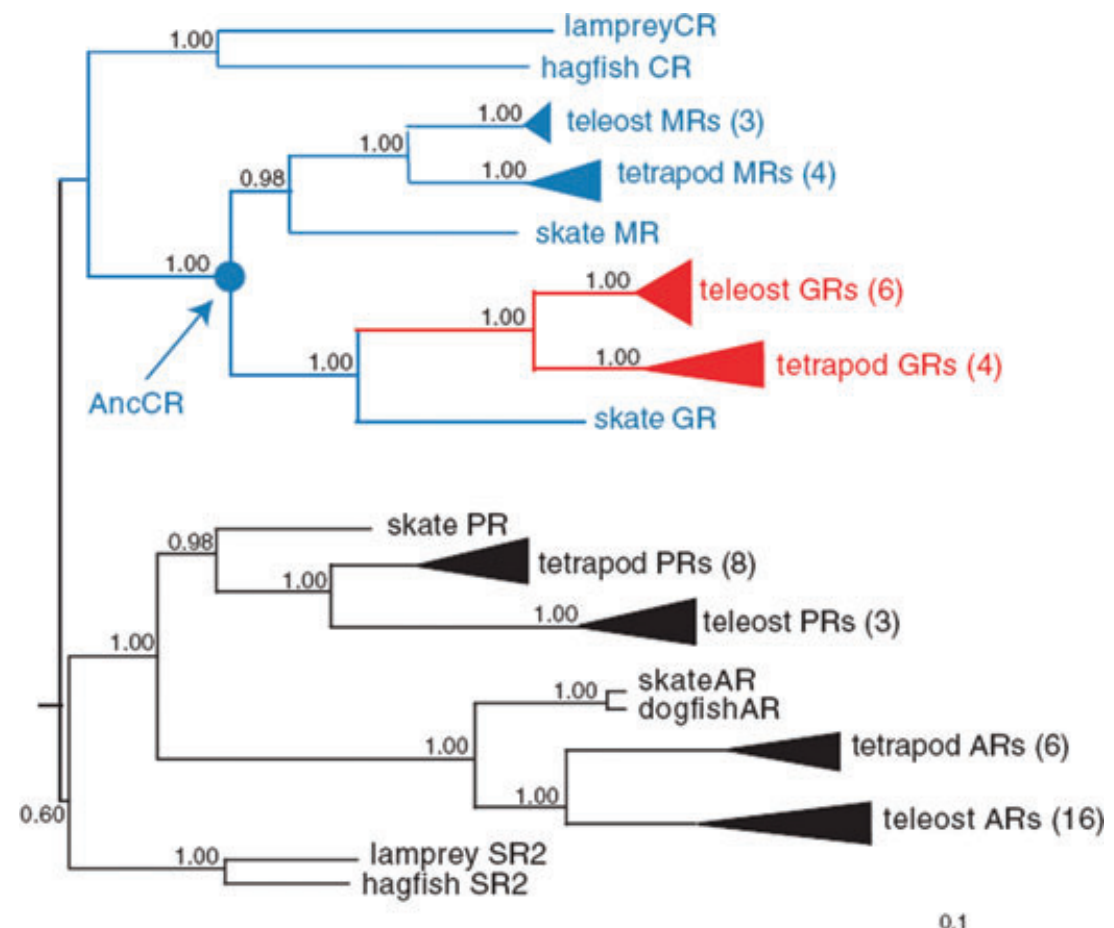

\begin{abstract}
Figure 1. Molecular phylogeny of steroid hormone receptors. Shown in parentheses are the number of sequences in each clade. The circle marks the ancestral corticoid receptor (AncCR) inferred from phylogenetic data. Reproduced from Ref. 16 with permission. (In color in Annals online.)
\end{abstract}

of the gnathastomes, an ancestral NR gene (derived from an earlier duplication of a gene most closely related to the modern estrogen receptor) duplicated to give rise to the progesterone receptor and CR lineages. A second largescale genome expansion that occurred in the gnathastome lineage approximately 450 million years ago gave rise to the GR and MR lineages (Fig. 1; Refs. 15, 16). The functional differentiation of these two receptors is proposed to have occurred by a "molecular exploitation" mechanism. The ancestral vertebrate $\mathrm{CR}$ is proposed to have had structural properties most similar to the MR, with highest affinity for 11-DOC and by chance for aldosterone (its capacity for biosynthesis did not appear until the evolution of the tetrapods through modification of $11 \beta$-hydroxylase; Refs. 16-18). Therefore, the ancestral CR and its descendants were structurally pre-adapted to bind aldosterone and thus "exploited" the lig- and, which appeared approximately 200 million years after the receptor. Bridgham and colleagues $^{16}$ suggest that as few as two amino acid substitutions were necessary in the GR lineage to generate a protein with high affinity for GCs but low affinity for aldosterone. The existence of two distinct hormone-receptor pairs allowed for the evolution of specific endocrine control of osmoregulation and the stress response.

Fully differentiated tissue capable of corticosteroidogenesis is found in extant chondrichthyean fishes, such as the holocephalans (ratfish) and elasmobranchs (sharks, skates, rays). The major corticosteroid in these species is 1-hydroxycorticosterone. ${ }^{19}$ In modern bony fishes (the teleosts), cortisol is the major steroid produced by adrenocortical tissue. ${ }^{14}$ In the majority of vertebrate species, the corticosteroidogenic tissue is separate from the chromaffin cells (producing catecholamines) and forms discrete 
organoids or organs referred to as interrenal glands. In most ray-finned fishes (the Teleosteii) the adrenocortical cells are embedded within the anterior portion of the kidney. In lobe-finned fishes (lungfishes; Dipnoi) cordlike masses of adrenocortical cells are found between the kidneys. Amphibians possess paired interrenal glands located on the ventral surface of the kidneys. It was not until the origin of the amniotes that corticosteroidogenic tissue became intermingled with chromaffin cells. In reptiles, chromaffin cells are intermingled with adrenocortical cells to varying degrees; however, the distinct adrenal zonation seen in mammals is only partly evident in reptiles and birds (reviewed by Norris ${ }^{14}$ ).

\section{Structural and Functional Evolution of the HPA Axis}

Neurosecretory neurons located in the preoptic/hypothalamic areas of vertebrates synthesize neuropeptides that control the HPA axis. CRF is the major neurohormone regulating the vertebrate HPA axis. ${ }^{20} \mathrm{CRF}$ is a 41 -amino acid peptide originally isolated from ovine hypothalamus and found to be the major hypothalamic releasing factor for pituitary corticotropin (ACTH; Ref. 21). The parvocellular subdivision of the paraventricular nucleus (PVN) is the major site of CRF synthesis in the mammalian hypothalamus. In nonmammalian species the hypophysiotropic CRF neurons are located in the anterior preoptic area (POA; preoptic nucleus). CRF is expressed outside of the hypothalamus in mammals in the limbic system (hippocampus, amygdala, nucleus accumbens, and bed nucleus of the stria terminalis [BNST]), hypothalamus, thalamus, cerebral cortex, cerebellum, and hindbrain. ${ }^{22}$ Similar distribution patterns of CRF neurons have been described in nonmammalian species. ${ }^{23,24}$ The conserved distribution pattern suggests ancient origins and strong positive selection on gene regulatory sequences that determine tissue-specific expression of CRF. ${ }^{25,26}$
The actions of CRF-like peptides are mediated by at least two $\mathrm{G}$ protein-coupled receptors $\left(\mathrm{CRF}_{1}\right.$ and $\left.\mathrm{CRF}_{2}\right)$ that have distinct tissue distributions and are known to mediate different and diverse actions of CRF peptides in the central nervous system and in the periphery. ${ }^{27}$ Orthologues of the mammalian $\mathrm{CRF}_{1}$ and $\mathrm{CRF}_{2}$ receptors have been isolated by molecular cloning from frogs, ${ }^{28,29}$ chicken, ${ }^{30,31}$ and fishes ${ }^{32-36}$; a third receptor was isolated from the catfish and is expressed in the pituitary and urophysis. ${ }^{32}$ Each CRF receptor gene gives rise to multiple splicing variants with distinct expression profiles in mammals, but similar splice variants of CRF receptors have yet to be identified in nonmammalian species. CRF binds to $\mathrm{CRF}_{1}$ receptors on pituitary corticotropes where it initiates signal transduction involving protein kinase A (PKA) and protein kinase $\mathrm{C}$ $(\mathrm{PKC})$ to increase secretion and biosynthesis of ACTH (reviewed in Ref. 26). ACTH stimulates corticosteroid biosynthesis by the adrenal cortex or interrenal gland. CRF and related peptides are also potent thyrotropin (TSH)releasing factors in nonmammalian species, acting via the $\mathrm{CRF}_{2}$ receptor expressed on thyrotrope cells. ${ }^{37,38}$ The actions of CRF-like peptides on TSH release may reflect an ancient role for these peptides in regulating life history transitions, such as amphibian metamorphosis, which is dependent on thyroid hormone (see Refs. 37, 39 for reviews).

Coincident with the discovery of CRF, structurally similar peptides were isolated from the caudal neurosecretory organ (urophysis) of several fishes (urotensin I; Ref. 40) and from the skin of the frog Phyllomedusa sauvageii (sauvagine; Ref. 41). At the time of their isolation it was thought that these peptides were orthologues of mammalian CRF, but subsequent analysis showed that fish and frogs possess orthologous GRF genes that are distinct from urotensin I and sauvagine. ${ }^{42,43}$ Subsequently, genes orthologous to urotensin I were isolated from mammalian species and named urocortin (now urocortin 1; Refs. 44, 45). While both CRF and urocortin 1 bind to and activate the $\mathrm{CRF}_{1}$ and 


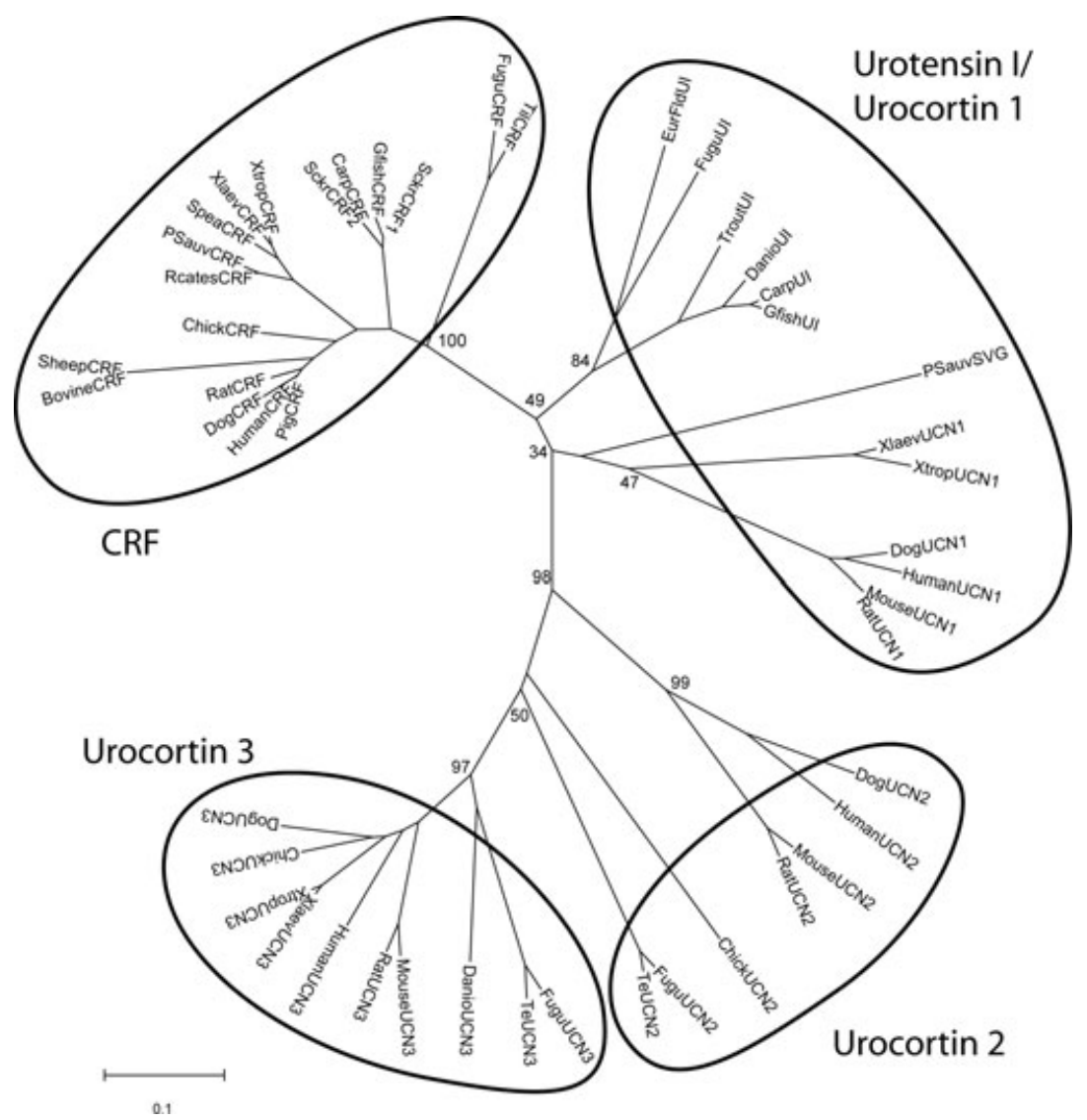

Figure 2. Neighbor joining tree of vertebrate corticotropin-releasing factor-like prohormone amino acid sequences. Abbreviations: CRF, corticotropin-releasing factor; Ul, urotensin I; SVG, sauvagine; UCN1, urocortin 1; UCN2, urocortin 2; UCN3, urocortin 3; Carp (Cyprinus carpio); Danio (Danio rerio, zebrafish); EurFld (Platichthys flesus, European flounder); Te (Tetraodon nigroviridis, pufferfish); Fugu (Takifugu rubripes, pufferfish); Gfish (Carassius auratus auratus, goldfish); Xlaev (Xenopus laevis, South African clawed frog); Xtrop (X. tropicalis); Rcates (Rana catesbeiana, North American bullfrog); Psauv (Phyllomedusa sauvageii); Sckr (Catostomus commersoni, sucker); Spea (Spea hammondii, Western spadefoot toad); Til (Tilapia mossambicus); Trout (Oncorhynchus mykiss). Modified from Ref. 46 with permission.

$\mathrm{CRF}_{2}$ receptors, $\mathrm{CRF}$ has higher affinity for the $\mathrm{CRF}_{1}$ receptor and urocortin 1 has higher affinity for the $\mathrm{CRF}_{2}$ receptor. ${ }^{27,28}$ Recently, we isolated a gene orthologous to mammalian urocortin 1 from Xenopus laevis. ${ }^{46}$ The frog urocortin 1 shares only $50 \%$ sequence similarity with sauvagine. A full-length sauvagine cDNA sequence isolated from the skin of $P$. sauvageii was recently deposited in GenBank (accession \#AY943910). Sauvagine appears to be a highly divergent urocortin 1 that may be specific to the Phyllomedusidae, and the relationship of sauvagine to other vertebrate urocortin genes remains to be determined.

Genomic analysis and molecular cloning recently led to the identification of two other CRF-like peptides in mammals that are selective for the $\mathrm{CRF}_{2}$ receptor; these are named urocortin 2 and urocortin $3 .{ }^{47-50}$ Urocortin 2 and 3 genes have also been identified in pufferfish, ${ }^{46}$ urocortin 2 in the chicken, and urocortin 3 in the frog $X$. laevis. ${ }^{46}$ Thus, in vertebrates there are at least four CRF paralogues (Fig. 2). The CRF family of peptides predates the 
chordates, with primitive functions in osmoregulation (see Refs. 8, 23 for reviews on the evolution of CRF-like peptides).

The bioactivity of CRF and related peptides is modulated by a secreted CRF-binding protein (CRF-BP) with affinity for CRF peptides that is similar to or greater than the affinity for the receptors. ${ }^{51}$ The CRF-BP was originally isolated and characterized from human liver and rat brain. ${ }^{52}$ Genes for the GRF-BP have been isolated and characterized and the expression patterns described in rat, mouse, sheep, frog ( $X$. laevis), fishes, and the honeybee. ${ }^{36,51,53-55}$ Also, we detected CRF-BP protein in brain extracts from sea lamprey, tilapia, turtle, and chicken, thus suggesting that the CRF-BP and its ability to bind CRF-like peptides was present in the earliest vertebrates. ${ }^{51}$

The major function of the CRF-BP appears to be to modulate access of CRF and related peptides to GRF receptors. ${ }^{56}$ The protein is expressed throughout the brain in vertebrates and overlaps with areas of expression of the ligands and CRF receptors and is thus positioned to modulate the bioavailability of CRF-like peptides. ${ }^{51}$ It is also widely expressed in peripheral tissues where it may influence interaction of CRF-like peptides with receptors. ${ }^{57}$ For example, CRF-BP is expressed in the human placenta and is secreted into the maternal circulation. ${ }^{58,59}$ Its expression level drops during late gestation while placental CRF expression increases, thus leading to increased free CRF in the circulation. This increased CRF bioavailability is hypothesized to play an important role in the timing of birth. ${ }^{60,61}$ Another example of peripherally expressed CRF-BP modulating CRF bioavailability is in the Xenopus tadpole tail where CRF, expressed by tail muscle cells, functions as an autocrine cytoprotective factor for tail muscle cell survival (that express $\mathrm{CRF}_{1}$; Ref. 62). The CRF-BP is expressed by cells between the muscles, connective tissue of the tail fin, and cells of the notochord sheath in the tadpole tail. ${ }^{63,64}$ Expression of the CRF-BP in tail is induced by thyroid hormone and it increases dramatically during spontaneous meta- morphosis. ${ }^{62}$ We showed that the CRF-BP negatively regulates bioavailability of CRF to its receptors on tail muscle cells. ${ }^{62}$ Thus, at metamorphic climax the induction of CRF-BP by thyroid hormone in tail promotes tail regression by modulating the cytoprotective actions of CRF.

While CRF plays an important role as a hypophysiotropin, CRF and urocortins are widely expressed in the brain and spinal cord in vertebrates where they function as neurotransmitters/neuromodulators to coordinate behavioral and autonomic responses to stress. ${ }^{23,57}$ For example, CRF-like peptides play important roles in the regulation of food intake, ${ }^{65,66}$ behavioral responses to stress, ${ }^{3,67-69}$ and influencing learning and memory consolidation. ${ }^{70-73}$ Recent findings show critical roles for the GRF system in energy homeostasis. ${ }^{74}$ CRF-like peptides, receptors, and binding protein are also expressed throughout the body where they are likely to influence most, if not all, physiological functions (Fig. 3; Ref. 57). The dysregulation of CRF and the HPA axis, at both the central and peripheral levels, has been implicated in the pathogenesis of human diseases, such as anxiety and depression, anorexia and obesity, inflammatory diseases, substance abuse, and preterm birth. ${ }^{75,76} \mathrm{CRF}$ and urocortins have some overlapping but many distinct roles in physiology and behavior. ${ }^{77}$ These distinctions are, in part, reflective of the differential expression and roles for the $\mathrm{CRF}_{1}$ and $\mathrm{CRF}_{2}$ receptors. ${ }^{27,57,68,69,78}$

\section{Stressor-dependent Activation of the HPA Axis}

The term stressor as used here is defined as any environmental change that elicits an increase in circulating corticosteroid concentration (i.e., a stress response). A variety of stressors (i.e., food deprivation, extreme weather, exercise, crowding, capture/confinement, shaking; Refs. 65, 79-82; see Fig. 4A) cause elevated plasma corticosteroid concentrations in different taxa. The increase in corticosteroid concentrations is associated with increased metabolic 


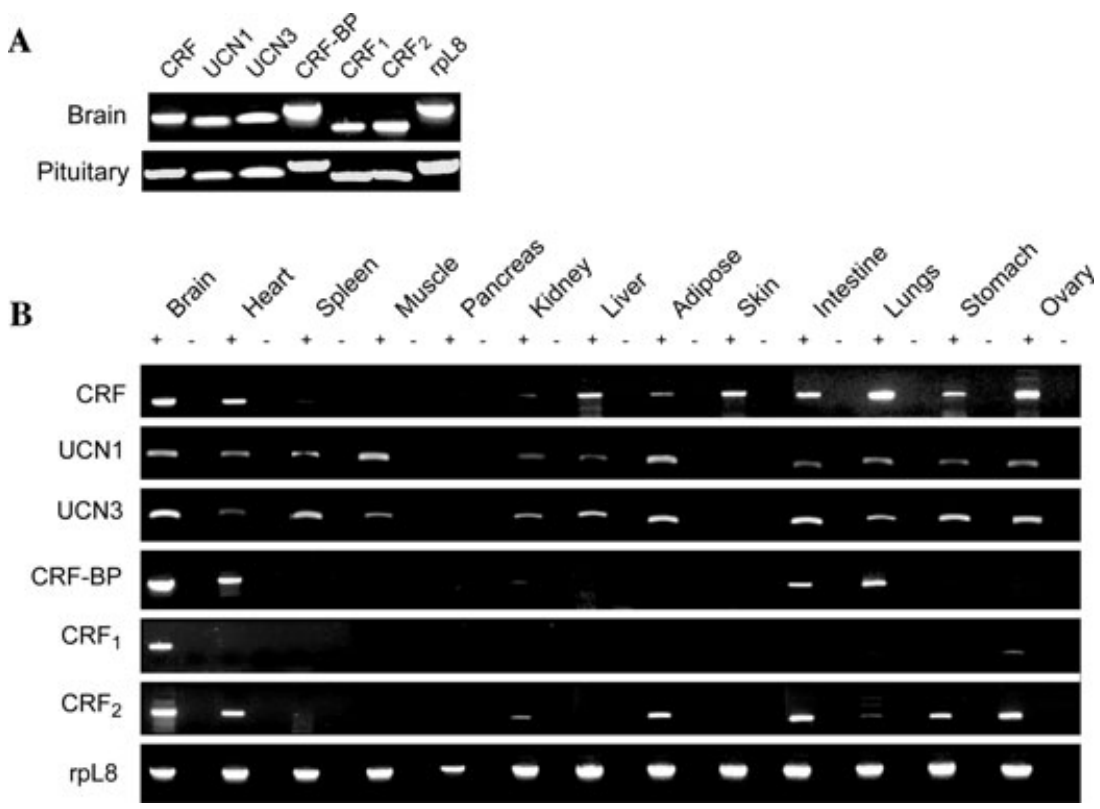

Figure 3. Tissue distribution of mRNAs for CRF, urocortins, CRF receptors, and CRF binding protein in juvenile $X$. laevis tissues analyzed by RT-PCR. (A) Expression analysis in brain and pituitary. (B) Expression analysis in diverse peripheral tissues. Abbreviations not listed in Fig. 2: CRF-BP, CRF binding protein; $C R F_{1}, C R F$ receptor 1; $C_{2 R F}, C R F$ receptor 2. Ribosomal L8 (rpL8) was used as a housekeeping gene to control for RNA quality and loading. Reproduced from Ref. 57 with permission.

A

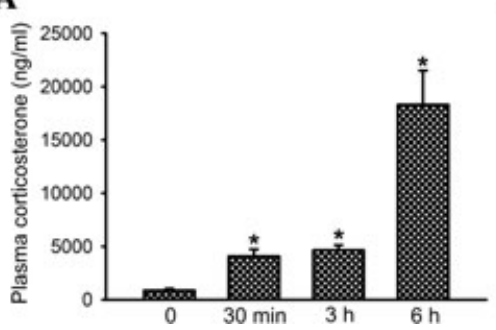

B

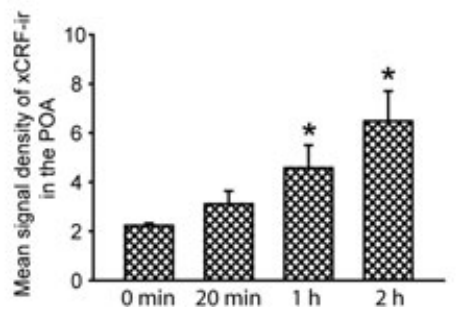

C

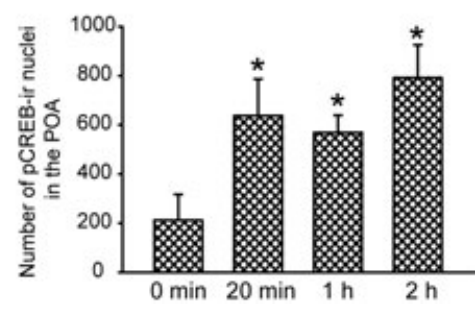

Figure 4. Changes in (A) plasma corticosterone concentration, (B) CRF immunoreactivity (CRF-ir; mean signal density), and (C) phosphorylated cAMP response element binding protein (CREB) immunoreactivity (CREB-ir; number of stained nuclei) in the anterior preoptic area of juvenile $X$. laevis exposed to shaking/confinement stressor for various times. Data presented are the mean + SEM. A significant difference from unstressed controls is denoted by * for $P<0.05$. Modified from Ref. $24(A)$ and Ref. 25 (B and $C)$ with permission.

rate and the mobilization of stored energy, which is thought to enhance performance during stressful events. ${ }^{79}$ While the immediate physiological response to a stressor results in the suppression of appetite owing to an increase in brain CRF, the elevation in circulating corticosteroids subsequently leads to increased food intake occurring several hours later. ${ }^{3,65,83}$ In- creases in plasma GCs exert feedback on the HPA axis to modulate the stress response (discussed below).

The key early event in the activation of the HPA axis by a stressor is the release of CRF from modified nerve terminals in the median eminence or the pituitary (teleosts). This increased secretion of CRF leads to a rise in 
plasma ACTH that in turn causes an increase in the production of GCs by adrenal cortical cells and a rise in plasma $\mathrm{GC}$ concentration. Rapid stressor-induced increases in CRF heteronuclear RNA (hnRNA; which reflects increased CRF gene transcription), CRF mRNA, and CRF peptide have been documented in rodents and frogs in POA/hypothalamic neurosecretory neurons (Fig. 4B; Ref. 26). In teleost fishes, exposure to stressors caused elevated CRF mRNA in the POA/hypothalamus and increased CRF concentration in the systemic circulation. ${ }^{26,67}$ These findings show that stressordependent activation of CRF genes arose early in vertebrate evolution and has been maintained by natural selection.

\section{Molecular Mechanisms of Stressor-dependent Activation of CRF Genes}

The conserved patterns of spatial, temporal, and stressor-dependent expression of CRF among species suggest that the cis regulatory elements responsible for the regulation of CRF genes arose early in vertebrate evolution. A comparative genomic analysis of vertebrate CRF genes identified several regions of strong sequence similarity. ${ }^{25,26}$ The proximal promoter regions (approximately $330 \mathrm{bp}$ upstream of the transcription start sites) share 94\% sequence similarity among human, rat, and ovine CRF genes ${ }^{84}$ and approximately $72 \%$ similarity among frog ( $x C R F b$ gene) and mammalian genes. While there is little or no sequence conservation in the introns among vertebrate classes, the $3^{\prime}$ untranslated regions (3' UTR) of tetrapods have several highly conserved stretches of nucleotide sequence (but the $3^{\prime}$ UTR of tetrapods is poorly conserved with fishes; M. Yao, The University of Michigan, Ann Arbor (Dept. of Molecular, Cellular and Developmental Biology), unpublished data). The sequence conservation in the $3^{\prime}$ UTR of tetrapods may indicate a role in translational control, which has received little atten- tion thus far, or to transcriptional control (e.g., perhaps tissue-specific expression).

Several intracellular signaling pathways are implicated in the regulation of CRF gene transcription. Perhaps the most significant pathway involves the activation of cAMPdependent PKA that leads to phosphorylation of the cAMP response element binding protein (CREB) (Fig. 4C; reviewed in Ref. 26). The transcriptional activity of CREB is regulated by phosphorylation at conserved serine residues, which occurs within minutes following exposure to stressors in mammals and frogs. ${ }^{26}$ Phosphorylation of CREB induces the recruitment of the co-activators CREB binding protein $(\mathrm{CBP})$ and p300 to gene promoters, leading to gene activation through increased histone acetylation. ${ }^{85,86}$ The cAMP activation of the CRF gene is explained by a highly conserved cAMP response element present in the proximal promoter of mammalian and frog genes. ${ }^{25}$ Mutational analysis has shown that this element is required for cAMP responsiveness of the $\mathrm{CRF}$ gene both in vitro and in vivo. ${ }^{25,87-89}$

Also implicated in the early transactivation of the CRF gene is the PKC pathway, acting through the transcription factors Fos and Jun (activator protein 1; AP-1). The AP-1 proteins are immediate early genes first identified based on their rapid and transient induction by growth factors. Both $c$-fos mRNA and protein have been shown to increase rapidly in several stress-related brain areas following exposure to stressors in mammals and frogs. ${ }^{26}$ Activation of the PKC pathway in cultured cells increased CRF promoter activity and gene expression. ${ }^{26}$ Evolutionarily conserved AP-1 binding sites are present in the $5^{\prime}$ flanking regions of vertebrate CRF genes. ${ }^{25,90}$ Two of these sites overlap with three putative glucocorticoid response element (GRE) sites, and it was shown that both AP-1 proteins and GR can specifically bind to this region of the human and frog CRF promoters. ${ }^{91,92}$ Mutational analysis supports that this region is important both for AP-1-mediated stimulation and for GR-mediated repression of CRF promoter activity. ${ }^{91}$ Several studies 
suggest that the early upregulation of CRF expression during the stress response does not depend on AP-1 binding proteins ${ }^{93-95}$; these authors concluded that while rapid phosphorylation of CREB is primarily responsible for the early induction of the $\mathrm{CRF}$ gene in the stress response, the PKG pathway may play a role in the delayed regulation of CRF expression in response to sustained or chronic stressors.

Nerve growth factor induced gene B (NGFIB; also termed Nur77) is an orphan nuclear receptor that is rapidly induced by exposure to stressors and implicated in the regulation of CRF gene expression. ${ }^{26}$ Consensus NGFI$\mathrm{B}$ binding sites (NGFI-B response element) are present in the rat and frog proximal CRF promoters. ${ }^{25,96}$ Activation of the PKA pathway in cultured cells rapidly increased NGFI-B expression and nuclear DNA binding activity of NGFI-B dimers, ${ }^{97}$ and cotransfection with an NGFI-B expression vector activated the CRF promoter. ${ }^{98,99}$ For a more thorough discussion of CREB, AP-1, and NGFI-B as well as other regulators of $\mathrm{CRF}$ gene expression, see the review by Yao and Denver. ${ }^{26}$

\section{Feedback Regulation of the HPA Axis by GCs}

The existence of negative feedback on HPA axis activity by circulating GCs is well established in mammals. While much less is known in nonmammalian species, the basic regulatory relationships within the HPA axis appear to be evolutionarily conserved. In mammals, CRF neurons located in the PVN of the hypothalamus and corticotropes of the anterior pituitary are negatively regulated by GGs, thereby limiting the magnitude and duration of the endocrine stress response. ${ }^{100-102}$ GCs act directly on hypothalamic CRF neurons to reduce CRF synthesis and secretion and on corticotropes in the anterior pituitary gland to decrease synthesis of POMC and secretion of ACTH. ${ }^{26,92}$ GCs also modulate the activity of PVN CRF neurons indirectly via descending pathways from the hippocampus, amygdala, and BNST or via pathways originating in the brain stem. ${ }^{2,102,103}$ While CRF neurons in the PVN are negatively regulated by GCs, other CRF-expressing cells are positively regulated. For example, GGs upregulate $\mathrm{CRF}$ expression in the central nucleus of the amygdala and BNST in rats and increase CRF expression in syncytiotrophoblast cells of the human placenta. ${ }^{26,92}$

In the frog $X$. laevis CRF expression is differentially regulated by GGs in discrete regions of the central nervous system. ${ }^{92}$ In the frog POA we found that GCs negatively regulated CRF primary transcript (hnRNA) and peptide. ${ }^{92} \mathrm{Us}_{\mathrm{s}}$ ing in vitro cell transfection, in vivo gene transfer, and in vitro DNA binding assays, we also showed that the frog proximal CRF promoters are negatively regulated by GGs and that a composite AP-1/GRE site implicated in the negative regulation of the human CRF gene is structurally and functionally conserved in the frog genes (X. laevis has two CRF genes owing to its tetraploidy; Ref. 43). Double-labeling immunofluorescent confocal microscopy showed that CRF and GR colocalize in cells of the frog POA. Similarly, in the rat, GRF and GR were found to co-localize in parvocellular neurons of the PVN. ${ }^{104-106}$ Negative regulation of CRF expression by GCis has also been reported in several teleost fishes, ${ }^{107-111}$ although the molecular mechanisms for this regulation have not been investigated. Thus, GGs acting via the GR can directly influence transcription of the CRF gene, and such regulation is phylogenetically ancient.

Indirect GC negative feedback on PVN neurons in mammals via a descending pathway originating in the hippocampus has been described in mammals. ${ }^{2,102,103}$ Cells in the hippocampus that express GR and MR send inhibitory projections to the PVN. ${ }^{102,112-114}$ Lesioning of the hippocampus resulted in increased CRF mRNA in the PVN and elevated circulating GCs, which led the authors to conclude that this brain structure exerts tonic repression on CRF expression in the PVN. ${ }^{12,115}$ The hippocampus appears to mediate PVN activity in response to psychological and 
multimodal stressors requiring higher order sensory processing. ${ }^{116,117}$

The amygdala has been shown to influence PVN CRF expression, and in contrast to the hippocampus, this pathway is primarily stimulatory. ${ }^{26}$ The CRF neurons in the amygdala and BNST are activated in response to fear/anxiety-provoking stressors. ${ }^{26}$ The BNST serves as a relay between the amygdala and the hypothalamus and is the major direct nonhypothalamic input to the parvocellular PVN. ${ }^{18,119}$ The CRF pathways originating in the amygdala and BNST are hypothesized to play a role in relaying stressor-dependent input to the hypothalamus and thereby facilitating PVN CRF release. ${ }^{120}$ In mammals, the central nucleus of the amygdala plays a key role in mediating neuroendocrine and behavioral response to stressors. ${ }^{114}$ In the frog, these functions may be subserved by the medial amygdala. ${ }^{24,92}$ Thus, the amygdala/BNST circuit is involved in relaying stimulatory signals to the PVN CRF neurons in response to certain types of psychological stressors, such as fear and anxiety. ${ }^{117,120}$

The sympathetic and parasympathetic branches of the autonomic nervous system centered in the brain stem control skeletal and smooth muscles of the vasculature, heart, gut, and other organs to coordinate with the endocrine and behavioral aspects of the stress response. Nuclei in the brain stem also mediate responses to physiological stress, arousal, and sensory processing. ${ }^{76,121,122}$ Studies in mammals suggest that basal CRF expression in the PVN is under tonic stimulatory control by brain stem structures. ${ }^{123,124}$

While comparable tract tracing and lesioning studies have not been conducted in nonmammalian species, our work in $X$. laevis supports that indirect modulation of hypothalamic GRF neurons by GCs via projections from limbic or brain stem structures is also present in frogs. For example, GRF is expressed in the amygdala, BNST, and medial pallium (homologue of the mammalian hippocampus) of the frog ${ }^{24,125}$ and its expression, and that of the immediate early gene $c$-fos are increased by exposure to a physical stressor. ${ }^{24}$ The frog amygdala, BNST, and medial pallium express GR, ${ }^{126}$ and GGs increased CRF in the amygdala and BNST (but decreased CRF in the POA; Ref. 92). Also, CRF is expressed in the locus coeruleus and other hindbrain structures in frogs. ${ }^{24}$ Thus, the limited data from nonmammalian species suggest that both direct and indirect GC feedback on hypophysiotropic CRF neurons is an ancient feature of the vertebrate HPA axis that has been evolutionarily conserved.

In addition to GC negative feedback, a GCindependent mechanism that limits CRF gene transcription immediately following exposure to an acute stressor has been described. ${ }^{127}$ Shepard and colleagues ${ }^{127}$ found that while CRF hnRNA was rapidly increased by restraint stress up to $30 \mathrm{~min}$, it returned to basal levels by 90 min in intact and adrenalectomized rats. Following exposure to the stressor, the expression of the repressor isoform of cAMP response element modulator (CREM), the inducible cAMP early repressor (ICER), was elevated in the rat PVN in a GC-independent manner, and by 3 h, CREM proteins were recruited to the CRF promoter. These authors proposed that, while GCs limit the sensitivity and magnitude of CRF transcriptional responses, the upregulation of ICER may play an important role in the immediate repression of CRF transcription following exposure to an acute stressor (reviewed in Ref. 128).

While genomic mechanisms of GC actions mediated by the GR and MR are the best studied, nongenomic actions mediated by $\mathrm{G}$ protein-coupled membrane receptors have also been shown. ${ }^{129-131}$ GCs caused rapid (within 15 min) inhibition of CRF-induced ACTH pituitary secretion, ${ }^{132-134}$ which could not be blocked by pretreatment with the GR antagonist RU486. ${ }^{135}$ GCs have also been shown to cause rapid inhibition of CRF release from rat hypothalamic explants in perifusion culture ${ }^{136}$ and to increase synaptic potentiation in the mouse hippocampus. ${ }^{137}$ Thus, in addition to 
the classical genomic pathways for GC action, rapid membrane-mediated signaling by GCs likely plays an important role in GC feedback on the HPA axis. ${ }^{6}$

\section{Summary and Future Directions}

The basic components and organization of the vertebrate neuroendocrine stress axis arose early in evolution and have been conserved. The high degree of conservation is attributable to the central adaptive role that this system plays in vertebrates. Central regulation of the axis depends on the actions of CRF, and CRF neurons are rapidly activated following exposure to a variety of stressors. The proximal promoter regions of $\mathrm{CRF}$ genes responsible for stressor-dependent activation are remarkably conserved across vertebrate species, but the regulatory regions responsible for tissuespecific expression have yet to be identified. The roles of the related peptides, the urocortins, in stress responses are beginning to be understood in mammals, but very little is known about their expression and function in nonmammalian species. Also, CRF and urocortins are expressed throughout the body where they may play diverse, but as yet poorly characterized, roles in tissue development and homeostasis. The actions of CRF-like peptides are mediated by two or more $G$ protein-coupled receptors and a secreted binding protein. Studies in mammals suggest distinct roles for CRF receptors and CRF-BP, but more research is needed to understand the diversity of functions of these proteins in vertebrates and their evolutionary history. The GCs are essential for feedback regulation of the stress axis and have a diversity of actions in development, physiology, and behavior that are partly understood in mammals but poorly known in nonmammalian species. Because of the deep evolutionary conservation of the neuroendocrine stress axis, nonmammalian species can serve as important model systems for elucidating the development, orga- nization, and function of the vertebrate stress axis.

\section{Acknowledgments}

Supported by National Science Foundation grants IBN 0235401 and IOS 0641587.

\section{Conflicts of Interest}

The author declares no conflicts of interest.

\section{References}

1. McCormick, S.D. \& D. Bradshaw. 2006. Hormonal control of salt and water balance in vertebrates. Gen. Comp. Endocrinol. 147: 3-8.

2. De Kloet, E.R. et al. 1998. Brain corticosteroid receptor balance in health and disease. Endocr. Rev. 19: 269-301.

3. Sapolsky, R.M., L.M. Romero \& A.U. Munck. 2000. How do glucocorticoids influence stress responses? Integrating permissive, suppressive, stimulatory, and preparative actions. Endocr. Rev. 21: 5589.

4. Dallman, M.F. et al. 2006. Glucocorticoids, chronic stress, and obesity. Prog. Brain. Res. 153: 75-105.

5. Pecoraro, N. et al. 2006. From Malthus to motive: how the HPA axis engineers the phenotype, yoking needs to wants. Prog. Neurobiol. 79: 247-340.

6. Tasker, J.G., S. Di \& R. Malcher-Lopes. 2006. Minireview: rapid glucocorticoid signaling via membrane-associated receptors. Endocrinology 147: 5549-5556.

7. Stefano, G.B. et al. 2002. The blueprint for stress can be found in invertebrates. Neuroendocrinol. Lett. 23: $85-93$.

8. Lovejoy, D.A. \& S. Jahan. 2006. Phylogeny of the corticotropin-releasing factor family of peptides in the metazoa. Gen. Comp. Endocrinol. 146: 1-8.

9. Denver, R.J. 1999. Evolution of the corticotropinreleasing hormone signaling system and its role in stress-induced phenotypic plasticity. Ann. N. Y. Acad. Sci. 897: 46-53.

10. Lopez-Bucio, J. et al. 2006. Novel signals for plant development. Current Opinion in Plant Biology 9: 523529.

11. Asami, T., T. Nakano \& S. Fujioka. 2005. Plant brassinosteroid hormones. Plant Horm. 72: 479504.

12. Li, J.M. \& H. Jin. 2007. Regulation of brassinosteroid signaling. Trends in Plant Science 12: 37-41. 
13. Thornton, J.W., E. Need \& D. Crews. 2003. Resurrecting the ancestral steroid receptor: ancient origin of estrogen signaling. Science 301: 1714-1717.

14. Norris, D. 2006. Vertebrate Endocrinology. Academic Press. New York.

15. Thornton, J.W. 2001. Evolution of vertebrate steroid receptors from an ancestral estrogen receptor by ligand exploitation and serial genome expansions. Proc. Natl. Acad. Sci. U.S.A. 98: 5671-5676.

16. Bridgham, J.T., S.M. Carroll \& J.W. Thornton. 2006. Evolution of hormone-receptor complexity by molecular exploitation. Science 312: 97-101.

17. Nunez, S. \& J.M. Trant. 1999. Regulation of interrenal gland steroidogenesis in the Atlantic stingray (Dasyatis sabina). F. Exp. Zool. 284: 517-525.

18. Jiang, J.Q. et al. 1998. Eel (Anguilla japonica) testis 11 beta-hydroxylase gene is expressed in interrenal tissue and its product lacks aldosterone synthesizing activity. Mol. Cell. Endocrinol. 146: 207-211.

19. Wendelaar Bonga, S.E. 1993. Endocrinology. In The Physiology of Fishes (CRC Series in Marine Science). D.H. Evans, Ed.: 469-502. CRC Press. Roca Raton, FL.

20. Vale, W., Vaughan, J. \& Perrin, M. 1997. Corticotropin-releasing factor (CRF) family of ligands and their receptors. The Endocrinologist 7(Suppl): S3-S9.

21. Vale, W. et al. 1981. Characterization of a 41amino acid residue ovine hypothalamic peptide that stimulates the secretion of corticotropin and B-endorphin. Science 213: 1394-1397.

22. Cummings, S. et al. 1983. Corticotropin-releasing factor immunoreactivity is widely distributed within the central nervous system of the rat: an immunohistochemical study. F. Neurosci. 3: 1355-1368.

23. Lovejoy, D.A. \& R.J. Balment. 1999. Evolution and physiology of the corticotropin-releasing factor (CRF) family of neuropeptides in vertebrates. Gen. Comp. Endocrinol. 115: 1-22.

24. Yao, M., N.J. Westphal \& R.J. Denver. 2004. Distribution and acute stressor-induced activation of corticotrophin-releasing hormone neurones in the central nervous system of Xenopus laevis. F. Neuroendocrinol. 16: 880-893.

25. Yao, M., M. Stenzel-Poore \& R.J. Denver. 2007. Structural and functional conservation of vertebrate corticotropin-releasing factor genes: evidence for a critical role for a conserved cyclic AMP response element. Endocrinology 148: 2518-2531.

26. Yao, M. \& R.J. Denver. 2007. Regulation of vertebrate corticotropin-releasing factor genes. Gen. Comp. Endocrinol. 153: 200-216.

27. Dautzenberg, F.M. \& R.L. Hauger. 2002. The GRF peptide family and their receptors: yet more partners discovered. Trends in Pharmacol. Sci. 23: 71-77.
28. Dautzenberg, F.M. et al. 1997. Identification of two corticotropin-releasing factor receptors from Xenopus laevis with high ligand selectivity: unusual pharmacology of the type 1 receptor. F. Neurochem. 69: 1640-1649.

29. Ito, Y. et al. 2006. Cloning and distribution of the bullfrog type 1 and type 2 corticotropin-releasing factor receptors. Gen. Comp. Endocrinol. 146: 291295.

30. De Groef, B. et al. 2003. Corticotropin-releasing hormone (GRH)-induced thyrotropin release is directly mediated through CRH receptor type 2 on thyrotropes. Endocrinology 144: 5537-5544.

31. Yu, J.X., L.Y. Xie \& A.B. AbouSamra. 1996. Molecular cloning of a type A chicken corticotropinreleasing factor receptor with high affinity for urotensin I. Endocrinology 137: 192-197.

32. Arai, M., I.Q. Assil \& A.B. Abou-Samra. 2001. Characterization of three corticotropin-releasing factor receptors in catfish: a novel third receptor is predominantly expressed in pituitary and urophysis. Endocrinology 142: 446-454.

33. Cardoso, J.C.R. et al. 2003. Isolation and characterisation of the corticotropin releasing factor receptor 1 (CRFR1) gene in a teleost fish, Fugu rubripes. DNA Seq. 14: 215-218.

34. Pohl, S. et al. 2001. Cloning and functional pharmacology of two corticotropin-releasing factor receptors from a teleost fish. Eur. F. Pharmacol. 430: 193-202.

35. Chen, C.C. \& R.D. Fernald. 2008. Sequences, expression patterns and regulation of the corticotropin-releasing factor system in a teleost. Gen. Comp. Endocrinol. 157: 148-155.

36. Huising, M. et al. 2004. Structural characterisation of a cyprinid (Cyprinus carpio L.) CRH, CRH-BP and CRH-R1, and the role of these proteins in the acute stress response. 7. Mol. Endocrinol. 32: 627648.

37. De Groef, B. et al. 2006. Role of corticotropinreleasing hormone as a thyrotropin-releasing factor in non-mammalian vertebrates. Gen. Comp. Endocrinol. 146: $62-68$.

38. Okada, R. et al. 2007. Involvement of the corticotropin-releasing factor (CRF) type 2 receptor in CRF-induced thyrotropin release by the amphibian pituitary gland. Gen. Comp. Endocrinol. 150: 437-444.

39. Denver, R.J., G.C. Boorse \& K.A. Glennemeier. 2002. Endocrinology of complex life cycles: amphibians. In Hormones, Brain and Behavior, Vol. 2. D. Pfaff et al., Eds.: 469-513. Academic Press, Inc., San Diego.

40. Lederis, K. et al. 1982. Complete amino acid sequence of urotensin I, a hypotensive and 
corticotropin-releasing neuropeptide from Catostomus.. Science 218: 162-165.

41. Montecucchi, P.C. \& A. Henschen. 1981. Amino acid composition and sequence analysis of sauvagine, a new active peptide from the skin of Phyllomedusa sauvagei. Int. F. Pept. Protein Res. 18: $113-$ 120.

42. Okawara, Y. et al. 1988. Cloning and sequence analysis of cDNA for corticotropin-releasing factor precursor from the teleost fish Catostomus commersoni. Proc. Natl. Acad. Sci. U.S.A. 85: 8439-8443.

43. Stenzel-Poore, M.P. et al. 1992. Characterization of the genomic corticotropin-releasing factor (CRF) gene from Xenopus laevis: two members of the CRF family exist in amphibians. Mol. Endocrinol. 6: 17161724.

44. Vaughan, J. et al. 1995. Urocortin, a mammalian neuropeptide related to fish urotensin I and to corticotropin-releasing factor. Nature 378: 287-292.

45. Donaldson, C.J. et al. 1996. Cloning and characterization of human urocortin. Endocrinology 137: 2167-2170.

46. Boorse, G.C. et al. 2005. Urocortins of the South African clawed frog, Xenopus laevis: conservation of structure and function in tetrapod evolution. $\mathrm{En}$ docrinology 146: 4851-4860.

47. Hsu, S.Y.H. \& A.J.W. 2001. Human stresscopin and stresscopin-related peptide are selective ligands for the type 2 corticotropin-releasing hormone receptor. Nature Medicine 7: 605-611.

48. Reyes, T.M. et al. 2001. Urocortin II: a member of the corticotropin-releasing factor (CRF) neuropeptide family that is selectively bound by type $2 \mathrm{CRF}$ receptors. Proc. Natl. Acad. Sci. U.S.A. 98: 28432848.

49. Lewis, K. et al. 2001. Identification of urocortin III, an additional member of the corticotropin-releasing factor (CRF) family with high affinity for the CRF2 receptor. Proc. Natl. Acad. Sci. U.S.A. 98: 7570-7575.

50. Hauger, R.L. et al. 2003. International union of pharmacology. XXXVI. Current status of the nomenclature for receptors for corticotropinreleasing factor and their ligands. Pharmacol. Rev. 55: $21-26$.

51. Seasholtz, A.F., Valverde, R.A. \& Denver, R.J. 2002. Corticotropin-releasing hormone-binding protein (CRH-BP): biochemistry and function from fishes to mammals. F. Endocrinol. 175: 89-97.

52. Potter, E. et al. 1991. Cloning and characterization of the cDNAs for human and rat corticotropin releasing factor binding proteins. Nature 349: $423-$ 426.

53. Alderman, S.L. \& N.J. Bernier. 2007. Localization of corticotropin-releasing factor, urotensin I, and CRF-binding protein gene expression in the brain of the zebrafish, Danio rerio. F. Comp. Neurol. 502: 783-793.

54. Alderman, S.L., J.C. Raine \& N.J. Bernier. 2008. Distribution and regional stressor-induced regulation of corticotrophin-releasing factor binding protein in rainbow trout (Oncorhynchus mykiss). F. Neuroendocrinol. 20: 347-358.

55. Huising, M.O. \& G. Flik. 2005. The remarkable conservation of corticotropin-releasing hormone $(\mathrm{CRH})$-binding protein in the honeybee (Apis mellifera) dates the CRH system to a common ancestor of insects and vertebrates. Endocrinology 146: 2165 2170 .

56. Seasholtz, A.F. et al. 2001. Mouse models of altered CRH-binding protein expression. Peptides 22: 743 751.

57. Boorse, G.C. \& R.J. Denver. 2006. Widespread tissue distribution and diverse functions of corticotropin-releasing factor and related peptides. Gen. Comp. Endocrinol. 146: 9-18.

58. Behan, D.P. et al. 1996. Measurement of corticotropin-releasing factor (CRF), CRF-binding protein (CRF-BP), and CRF/CRF-BP complex in human plasma by two-site enzyme-linked immunoabsorbant assay. 7. Clin. Endocrinol. Metab. 81: 2579-2586.

59. Linton, E. et al. 1993. Corticotropin releasing hormone-binding protein (CRH-BP) plasma levels decrease during the third trimester of normal pregnancy. 7. Clin. Endocrinol. Metab. 76: 260-262.

60. McLean, M. \& R. Smith. 2001. Corticotrophinreleasing hormone and human parturition. Reproduction 121: 493-501.

61. Hillhouse, E.W. \& D.K. Grammatopoulos. 2002. Role of stress peptides during human pregnancy and labour. Reproduction 124: 323-329.

62. Boorse, G.C. et al. 2006. Corticotropin-releasing factor is cytoprotective in Xenopus tadpole tail: Coordination of ligand, receptor, and binding protein in tail muscle cell survival. Endocrinology 147: 14981507.

63. Berry, D.L. et al. 1998. The expression pattern of thyroid hormone response genes in remodeling tadpole tissues defines distinct growth and resorption gene expression programs. Dev. Biol. 203: 24 35.

64. Berry, D.L., R.A. Schwartzman \& D.D. Brown. 1998. The expression pattern of thyroid hormone response genes in the tadpole tail identifies multiple resorption programs. Dev. Biol. 203: 12 23.

65. Crespi, E.J. \& R.J. Denver. 2005. Roles of stress hormones in food intake regulation in anuran amphibians throughout the life cycle. Comp. Biochem. E Physiol. A-Comp. Physiol. 141: 381-390. 
66. Mastorakos, G. \& E. Zapanti. 2004. Thy hypothalamic-pituitary-adrenal axis in the neuroendocrine regulation of food intake and obesity: The role of corticotropin releasing hormone. Nutritional Neuroscience. 7: 271-280.

67. Flik, G. et al. 2006. CRF and stress in fish. Gen. Comp. Endocrinol. 146: 36-44.

68. Bale, T.L. et al. 2002. Mice deficient for both corticotropin-releasing factor receptor 1 (CRFR1) and CRFR2 have an impaired stress response and display sexually dichotomous anxiety-like behavior. 7. Neurosci. 22: 193-199.

69. Bale, T.L. \& W.W. Vale. 2004. CRF and CRF receptors: Role in stress responsivity and other behaviors. Ann. Rev. Pharmacol. E Tox. 44: 525-557.

70. Fenoglio, K.A., K.L. Brunson \& T.Z. Baram. 2006. Hippocampal neuroplasticity induced by early-life stress: functional and molecular aspects. Front. Neuroendocrinol. 27: 180-192.

71. Gulpinar, M.A. \& B.C. Yegen. 2004. The physiology of learning and memory: role of peptides and stress. Curr. Protein E Peptide Sci. 5: 457-473.

72. Roozendaal, B., G. Schelling \& J.L. McGaugh. 2008. Corticotropin-releasing factor in the basolateral amygdala enhances memory consolidation via an interaction with the beta-adrenoceptor-cAMP pathway: dependence on glucocorticoid receptor activation. F. Neurosci. 28: 6642-6651.

73. Todorovic, C. et al. 2007. Differential activation of CRF receptor subtypes removes stress-induced memory deficit and anxiety. European f. Neurosci. 25: 3385-3397.

74. Carlin, K.M., W.W. Vale \& T.L. Bale. 2006. Vital functions of corticotropin-releasing factor (CRF) pathways in maintenance and regulation of energy homeostasis. Proc. Natl. Acad. Sci. U.S.A. 103: 3462 3467.

75. Majzoub, J.A. et al. 1999. A central theory of preterm and term labor: putative role for corticotropin-releasing hormone. Am. F. Obst. $\&$ Gynecol. 180: S232-S241.

76. Tsigos, C. \& G.P. Chrousos. 2002. Hypothalamicpituitary-adrenal axis, neuroendocrine factors and stress. F. Psychosomat. Res. 53: 865-871.

77. Fekete, E.M. \& E.P. Zorrilla. 2007. Physiology, pharmacology, and therapeutic relevance of urocortins in mammals: ancient CRF paralogs. Front. Neuroendocrinol. 28: 1-27.

78. Rissman, R.A. et al. 2007. Corticotropin-releasing factor receptors differentially regulate stressinduced tau phosphorylation. 7. Neurosci. 27: 6552 6562.

79. Wingfield, J.C. et al. 1998. Ecological bases of hormone-behavior interactions: the "emergency life history stage". Am. Zool. 38: 191-206.
80. Sapolsky, R.M. 2000. Stress hormones: good and bad. Neurobiol. Dis. 7: 540-542.

81. Licht, P., B.R. McCreery, R. Barnes, \& R. Pang. 1983. Seasonal and stress related changes in plasma gonadotropins, sex steroids, and corticosterone in the bullfrog, Rana catesbeiana. Gen. Comp. Endocrinol. 50: $124-145$.

82. Zerani, M., F. Amabili, G. Mosconi, \& A. Gobbetti. 1991. Effects of captivity stress on plasma steroidlevels in the green frog, Rana esculenta, during the annual reproductive cycle. Comp. Biochem. E Physiol A-Comp. Physiol. 98: 491-496.

83. Crespi, E.J. \& R.J. Denver. 2004. Ontogeny of corticotropin-releasing factor effects on locomotion and foraging in the Western spadefoot toad (Spea hammondii). Horm. Behav. 46: 399-410.

84. Vamvakopoulos, N.C. et al. 1990. Structural analysis of the regulatory region of the human corticotropin releasing hormone gene. Febs Letters. 267: 1-5.

85. Johannessen, M. et al. 2004. Synergistic activation of CREB-mediated transcription by forskolin and phorbol ester requires PKC and depends on the glutamine-rich Q2 transactivation domain. Cellular Signalling. 16: 1187-1199.

86. Mayr, B. \& M. Montminy. 2001. Transcriptional regulation by the phosphorylation-dependent factor CREB. Nature Rev. Mol. Cell Biol. 2: 599 609.

87. Seasholtz, A.F., R.C. Thompson \& J.O. Douglass. 1988. Identification of a cyclic adenosine monophosphate-responsive element in the rat corticotropin-releasing hormone gene. Mol. Endocrinol. 2: 1311-1319.

88. Spengler, D. et al. 1992. Identification and characterization of a $3^{\prime}, 5^{\prime}$-cyclic adenosine monophosphate-responsive element in the human corticotropin-releasing hormone gene promoter. Mol. Endocrinol. 6: 1931-1941.

89. Nikodemova, M. et al. 2003. Cyclic adenosine $3^{\prime}, 5^{\prime}$-monophosphate regulation of corticotropinreleasing hormone promoter activity in AtT-20 cells and in a transformed hypothalamic cell line. Endocrinology 144: 1292-1300.

90. Vamvakopoulos, N.C. \& G.P. Chrousos. 1994. Hormonal-regulation of human corticotropinreleasing hormone gene-expression -implications for the stress-response and immune/inflammatory reaction. Endocr. Rev. 15: 409-420.

91. Malkoski, S.P. \& R.I. Dorin. 1999. Composite glucocorticoid regulation at a functionally defined negative glucocorticoid response element of the human corticotropin-releasing hormone gene. Mol. Endocrinol. 13: 1629-1644.

92. Yao, M., J. Schulkin \& R.J. Denver. 2008. Evolutionarily conserved glucocorticoid regulation of 
corticotropin-releasing factor expression. Endocrinology 149: 2352-2360

93. Kovacs, K.J. \& P.E. Sawchenko. 1996. Sequence of stress-induced alterations in indices of synaptic and transcriptional activation in parvocellular neurosecretory neurons. F. Neurosci. 16: 262-273.

94. Kovacs, K.J. \& P.E. Sawchenko. 1996. Regulation of stress-induced transcriptional changes in the hypothalamic neurosecretory neurons. 7. Mol. Neurosci. 7: 125-133.

95. Kovacs, K.J., C. Arias \& P.E. Sawchenko. 1998. Protein synthesis blockade differentially affects the stress-induced transcriptional activation of neuropeptide genes in parvocellular neurosecretory neurons. Brain Res. Mol. Brain Res. 54: 85-91.

96. Wilson, T.E. et al. 1991. Identification of the DNAbinding site for NGFI-B by genetic selection in yeast. Science 252: 1296-1300.

97. Maira, M. et al. 2003. Dimer-specific potentiation of NGFI-B (Nur77) transcriptional activity by the protein kinase a pathway and AF-1-dependent coactivator recruitment. Mol. Cell. Biol. 23: 763-776.

98. Murphy, E.P. \& O.M. Conneely. 1997. Neuroendocrine regulation of the hypothalamic pituitary adrenal axis by the nurr $1 /$ nur77 subfamily of nuclear receptors. Mol. Endocrinol. 11: 39-47.

99. Kovalovsky, D. et al. 2002. Activation and induction of NUR77/NURR1 in corticotrophs by $\mathrm{CRH} / \mathrm{cAMP}$ : involvement of calcium, protein kinase A, and MAPK pathways. Mol. Endocrinol. 16: 1638-1651.

100. Dallman, M.F. et al. 1992. Stress, feedback and facilitation in the hypothalamo-pituitary-adrenal axis. $\mathcal{F}$. Neuroendocrinol. 4: 517-526.

101. Whitnall, M.H. 1993. Regulation of the hypothalamic corticotropin-releasing hormone neurosecretory system. Prog. Neurobiol. 40: 573-629.

102. Makino, S., K. Hashimoto \& P.W. Gold. 2002. Multiple feedback mechanisms activating corticotropinreleasing hormone system in the brain during stress. Pharmacol. Biochem. Behav. 73: 147-158.

103. Schulkin, J., P.W. Gold \& B.S. McEwen. 1998. Induction of corticotropin-releasing hormone gene expression by glucocorticoids: implication for understanding the states of fear and anxiety and allostatic load. Psychoneuroendocrinology 23: 219-243.

104. Ceccatelli, S. et al. 1989. Coexistence of glucocorticoid receptor-like immunoreactivity with neuropeptides in the hypothalamic paraventricular nucleus. Exp. Brain Res. 78: 33-42.

105. Cintra, A. et al. 1987. Presence of glucocorticoid receptor immunoreactivity in corticotrophin releasing factor and in growth hormone releasing factor immunoreactive neurons of the rat di- and telencephalon. Neurosci. Lett. 77: 25-30.
106. Cintra, A. et al. 1991. Central peptidergic neurons as targets for glucocorticoid action: evidence for the presence of glucocorticoid receptor immunoreactivity in various types of classes of peptidergic neurons. F. Steroid Biochem. Mol. Biol. 40: 93 103.

107. Bernier, N.J., N. Bedard \& R.E. Peter. 2004. Effects of cortisol on food intake, growth, and forebrain neuropeptide $\mathrm{Y}$ and corticotropin-releasing factor gene expression in goldfish. Gen. Comp. Endocrinol. 135: $230-240$.

108. Bernier, N.J., X.W. Lin \& R.E. Peter. 1999. Differential expression of corticotropin-releasing factor (CRF) and urotensin I precursor genes, and evidence of CRF gene expression regulated by cortisol in goldfish brain. Gen. Comp. Endocrinol. 116: 461477.

109. Morley, S.D. et al. 1991. Corticotropin-releasing factor (CRF) gene family in the brain of the teleost fish Catostomus commersoni (white sucker): molecular analysis predicts distinct precursors for two CRFs and one urotensin I peptide. Mol. Mar. Biol. Biotechnol. 1: 48-57.

110. Olivereau, M. \& J. Olivereau. 1990. Effect of pharmacological adrenalectomy on corticotropinreleasing factor-like and arginine vasotocin immunoreactivities in the brain and pituitary of the eel: immunocytochemical study. Gen. Comp. Endocrinol. 80: $199-215$.

111. Fryer, J.N. \& G. Boudreault-Chateauvert. 1981. Cytological evidence for activation of neuroendocrine cells in the parvocellular preoptic nucleus of the goldfish hypothalamus following pharmacological adrenalectomy. Cell and Tissue Res. 218: 129 140.

112. Sapolsky, R.M., S. Zolamorgan \& L.R. Squire. 1991. Inhibition of glucocorticoid secretion by the hippocampal formation in the primate. 7 . Neurosci. 11: 3695-3704.

113. Herman, J.P. et al. 2003. Central mechanisms of stress integration: hierarchical circuitry controlling hypothalamo-pituitary-adrenocortical responsiveness. Front. Neuroendocrinol. 24: 151-180.

114. Herman, J.P. et al. 2005. Limbic system mechanisms of stress regulation: hypothalamo-pituitaryadrenocortical axis. Prog Neuropsychopharmacol. Biol. Psychiatry 29: 1201-1213.

115. Herman, J.P. et al. 1989. Evidence for hippocampal regulation of neuro-endocrine neurons of the hypothalamo pituitary adrenocortical axis. f. Neurosci. 9: $3072-3082$.

116. Jacobson, L. \& R. Sapolsky. 1991. The role of the hippocampus in feedback-regulation of the hypothalamic-pituitary-adrenocortical axis. Endocr. Rev. 12: 118-134. 
117. Herman, J.P. \& W.E. Cullinan. 1997. Neurocircuitry of stress: central control of the hypothalamopituitary-adrenocortical axis. Trends Neurosci. 20: 78-84.

118. Gunningham, E.T., M.C. Bohn \& P.E. Sawchenko. 1990. Organization of adrenergic inputs to the paraventricular and supraoptic nuclei of the hypothalamus in the rat. F. Comp. Neurol. 292: 651-667.

119. Sawchenko, P.E. \& L.W. Swanson. 1983. The organization and biochemical specificity of afferent projections to the paraventricular and supraoptic nuclei. Prog. Brain Res. 60: 19-29.

120. Gray, T.S. 1993. Amygdaloid CRF pathways: role in autonomic, neuroendocrine, and behavioral responses to stress. Ann. N. Y. Acad. Sci. 697: 53-60.

121. Brunson, K.L. et al. 2001. Neurobiology of the stress response early in life: evolution of a concept and the role of corticotropin releasing hormone. Mol. Psychiatr. 6: 647-656.

122. Ziegler, D.R. \& J.P. Herman. 2002. Neurocircuitry of stress integration: anatomical pathways regulating the hypothalamo-pituitary-adrenocortical axis of the rat. Integr. Comp. Biol. 42: 541-551.

123. Kiss, A., M. Palkovits \& G. Aguilera. 1996. Neural regulation of corticotropin releasing hormone (CRH) and CRH receptor mRNA in the hypothalamic paraventricular nucleus in the rat. F. Neuroendocrinol. 8: 103-112.

124. Ziegler, D.R., W.A. Cass \& J.P. Herman. 1999. Excitatory influence of the locus coeruleus in hypothalamic-pituitary-adrenocortical axis responses to stress. F. Neuroendocrinol. 11: 361369.

125. Tonon, M.C. et al. 1985. Immunohistochemical localization and radioimmunoassay of corticotropinreleasing factor in the forebrain and hypophysis of the frog Rana ridibunda. Neuroendocrinology 40: 109 119.

126. Yao, M., F. Hu \& R.J. Denver. 2008. Distribution and corticosteroid regulation of glucocorticoid receptor in the brain of Xenopus laevis. F. Comp. Neurol. 508: $967-982$.
127. Shepard, J.D. et al. 2005. Role of glucocorticoids and cAMP-mediated repression in limiting corticotropin-releasing hormone transcription during stress. F. Neurosci. 25: 4073-4081.

128. Aguilera, G. et al. 2007. Negative regulation of corticotropin releasing factor expression and limitation of stress response. Stress 10: 153-161.

129. Chen, Y.-Z. \& J. Qiu. 1999. Pleiotropic signaling pathways in rapid, nongenomic action of glucocorticoid. Mol. Cell Biol. Res. Comm. 2: 145-149.

130. Borski, R.J. 2000. Nongenomic membrane actions of glucocorticoids in vertebrates. Trends Endocrinol. Metab. 11: 427-436.

131. Norman, A.W., M.T. Mizwicki \& D.P.G. Norman. 2004. Steroid-hormone rapid actions, membrane receptors and a conformational ensemble model. Nature Rev. Drug Discov. 3: 27-41.

132. Widmaier, E.P. \& M.F. Dallman. 1984. The effects of corticotropin-releasing factor on adrenocorticotropin secretion from perifused pituitaries in vitro: rapid inhibition by glucocorticoids. Endocrinology 115: 2368-2374.

133. Bilezikjian, L.M. \& W.W. Vale. 1983. Glucocorticoids inhibit corticotropin-releasing factor-induced production of adenosine-3',5'-monophosphate in cultured anterior pituitary cells. Endocrinology 113: 657-662.

134. Iwasaki, Y. et al. 1997. Non-genomic mechanisms of glucocorticoid inhibition of adrenocorticotropin secretion: possible involvement of GTP-binding protein. Biochem. Biophys. Res. Commun. 235: 295-299.

135. Hinz, B. \& R. Hirschelmann. 2000. Rapid nongenomic feedback effects of glucocorticoids on GRF-induced ACTH secretion in rats. Pharm. Res. 17: $1273-1277$.

136. Suda, T. et al. 1985. In vitro study of immunoreactive corticotropin-releasing factor release from the rat hypothalamus. Life Sci. 37: 1499-1505.

137. Wiegert, O., M. Joels \& H. Krugers. 2006. Timing is essential for rapid effects of corticosterone on synaptic potentiation in the mouse hippocampus. Learn. Mem. 13: 110-113. 